\title{
Integration of Spatial User-Item Relations into Recommender Systems
}

\author{
Carsten Stiller, Fred Roß and Christoph Ament \\ Ilmenau University of Technology \\ Systems Analysis Group \\ PO Box 100565, 98684 Ilmenau, Germany
}

\begin{abstract}
Recommender systems aggregate information about users and items to be recommended to generate adequate recommendations. This paper proposes two approaches to include information about spatial relationships of users and items in order to improve the quality of recommendations. The two approaches are compared with non-spatial recommendation using two synthesized data sets and four different evaluation metrics. The results show how spatial information can improve recommender results. Furthermore each of the two approaches can perform better than the other, depending on what assumption of user behaviour is represented by the data set. Special ramp-up problems, occurring when including spatial information into recommender systems and different application areas for spatial recommender systems are discussed.
\end{abstract}

\section{Introduction}

Most applications of recommender systems are based in online domains like recommendations of articles in online shopping systems, news or scientific publications. In a lot of the remaining applications the recommendations are more or less independent from spatial context as well. For instance movie recommendations are to a high degree independent from location, as movies can be seen nearly everywhere in cinemas, TV or bought or rented in DVD stores.

Given this background it is somewhat understandable that spatial relation has not often been considered as valuable information in recommender systems. But with increasing adoption of such systems into other domains it is more and more reasonable to have a closer look at different contextual information to add new methods to the recommender system tool box.

The remaining part of this paper is organised as follows: The next section introduces our approaches to add spatial awareness to known recommender system techniques. Our approach to evaluate the quality of recommendation results is described in section 3, the according results are summarised in section 4 and discussed in section 5. Ramp-up problems in this kind of systems are considered in section 6 , an overview of related work is given in section 7 . Sections 8 and 9 end this paper with an outline of future works on this topic within our research group and a conclusion.

\section{Extension of known Recommender Systems}

In order to incorporate spatial information into recommender systems we propose two extensions to well known recommender systems. Although the approaches are shown only for one distinct recommendation algorithm they can be transferred to other algorithms based on user similarity as well.

\subsection{Non-Spatial Recommender Systems}

The task of a recommender system is to select the item(s) out of a large set of items $I$ which the user does not know but will most likely like and/or purchase. To do so it predicts the rating $\hat{r}(u, i)$ of an item $i$ for the current user $u$ by comparing it with ratings of users with similar tastes or decision behavior out of a set of users $U$. User-based collaborative filtering approaches for this prediction use similarity patterns in past ratings of the users to determine the similarity between the users. The sparse matrix $R$ of size $\operatorname{dim}(U) \times \operatorname{dim}(I)$ contains these past ratings and a certain rating can be addressed as $r(u, i)=R_{u, i}$, where $r(u, i)$ might be empty.

With this information the predicted rating is in most cases calculated by a function similar to

$$
\hat{r}(u, i)=w \cdot \sum_{\substack{k \in U \\ k \neq u}} \operatorname{sim}(u, k) \cdot r(k, i),
$$


where

$$
w=\frac{1}{\sum_{\substack{k \in U \\ k \neq u}} \operatorname{sim}(u, k)}
$$

is a weighting factor and $\operatorname{sim}(u, k)$ is a weight of similarity between user $u$ and $k$. Common functions used to describe similarity are correlation or cosine-based vector similarity over the vector of items both users have rated [2].

Other approaches to predict ratings include item-based filtering which incorporates meta-data about the items into the prediction. But as this methods are not important for the approach proposed in this paper they will not be discussed in detail here.

\subsection{Spatial Approach 1}

When information about the location of the users and of the items are available, the rating prediction can be adapted using the assumption that items are more interesting if users who have to come a long way to the item rank them high. One easy way to incorporate this information is to estimate the variance of the distribution of users out of $U$ who rated item $i$ around the item and to compare it with the distance of the current user and this item. The variance can be estimated by

$$
\sigma_{i}^{2}=\frac{1}{k_{i}} \sum_{\substack{k \in U \\ k \neq u}}\left\|x_{i}-x_{k}\right\|_{2}^{2},
$$

where $x_{i}$ and $x_{k}$ are the locations of the item $i$ and the user $k$ respectively, while $\left\|x_{i}-x_{k}\right\|_{2}$ is the euclidean distance between both locations. $k_{i}$ is the number of users that rated item $i$. Further equation (1) is modified to

$$
\hat{r}(u, i)=w_{d} \cdot \sum_{\substack{k \in U \\ k \neq u}} \operatorname{sim}(u, k) \cdot r(k, i)
$$

with the weight $w_{d}$ calculated as

$$
w_{d}=w \cdot \exp \left(-\frac{1}{2} \cdot \frac{\left\|x_{i}-x_{u}\right\|_{2}^{2}}{\sigma_{i}^{2}}\right),
$$

where $x_{u}$ is the location of the current user. This algorithm does not distinguish between high or low ratings of the users but only weights the result of equation (1), which already includes these ratings.

\subsection{Spatial Approach 2}

The drawback of the approach just described is its inability to reflect different behaviour within different groups of users. This can be done by weighting every user rating according to the distance of each of the other users to the item in comparison to the current user. The equation

$$
w_{s}(u, k, i)=\exp \left(-\frac{1}{2} \cdot\left(\frac{\left\|x_{i}-x_{u}\right\|_{2}}{\left\|x_{i}-x_{k}\right\|_{2}}\right)^{2}\right)
$$

is used to depict this weighting. To use this equation, equation (1) is modified to

$$
\hat{r}(u, i)=w \cdot \sum_{\substack{k \in U \\ k \neq u}} \operatorname{sim}(u, k) \cdot w_{s}(u, k, i) \cdot r(k, i),
$$

weighted by

$$
w=\frac{1}{\sum_{\substack{k \in U \\ k \neq u}} \operatorname{sim}(u, k) \cdot w_{s}(u, k, i)} .
$$

According to the classification of hybrid recommender systems, given by Burke in [6], both approaches would classify as Feature Combination hybrids.

\section{Evaluation}

Evaluation of recommender algorithms can be difficult as most algorithms are designed to adapt to certain user behaviour and show different results according to the data set and performance metric used. Therefore it is important to set up an evaluation strategy fitted to the needs of the aimed field of application.

\subsection{Data Sets}

As summarised by Herlocker et al. [8] and naturally comprehensible, the best way to evaluate recommender systems is to use real world data sets from the domain of intended application. But as we are in an early stage of developing spatial aware recommender systems we could not yet extract data sets from our application domain. Existing data sets used for benchmarking recommender systems mostly lack spatial information or the spatial information is not applicable to our problem (as in the Chicago Entree restaurant recommender usage data set of Burke available at [3].)

On the other hand, Herlocker et al. also state that "it may be appropriate to first evaluate algorithms against synthetic data sets to identify the promising ones for further study", as long as there is enough knowledge about the user preferences in the applications domain.

The aimed user group within our project are elderly persons with a broad range of personal interests and personal abilities. For this user group, services from daily live, care and health are to be recommended to overcome possible information deficits or to improve existing channels of information. At the moment first user surveys were conducted 

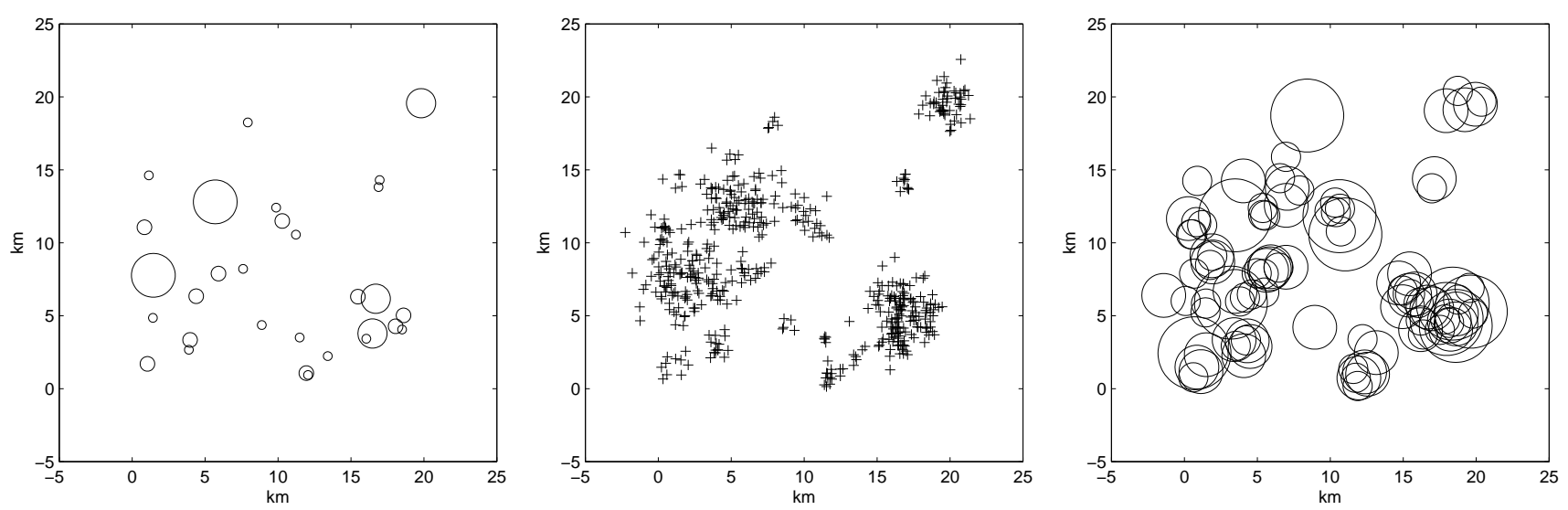

Figure 1. Graphical representation of the used data sets: a) cities, circles represent standard derivation, b) users, c) services, circles represent standard variation as used in data set 1, diameter reduced to $\frac{1}{2}$ for clarity.

(see [11]) and first results show that personal mobility is a strong influencing factor for grouping users. This implies that distance is important when selecting a service.

Therefore we generated data sets which include different locations (the centers of "cities" or "neighbourhoods") around which users and services are located. In a square area with edge length of $20 \mathrm{~km}, 30$ cities were randomly placed. Services and users are normal distributed around those city centres with different standard derivations. Two cities had a standard derivation of $1.5 \mathrm{~km}$ around the city centres, additionally three cities with $1 \mathrm{~km}, 10$ cities with $0.5 \mathrm{~km}$ and 15 cities with $0.3 \mathrm{~km}$ derivation where added. The placement of the cities and their standard variation can be seen in Figure 1 a). In each city of this four groups 100, 50, 10 and 5 users respectively where placed, making up a total of 525 users as depicted in Figure $1 \mathrm{~b}$ ). On the other hand $10,5,5$ or 1 services in these cities make up a total of 100 services. The placement of the services can be seen in Figure $1 \mathrm{c})$.

To see, how the algorithms perform when different user behaviour is given, two sets of user ratings here created:

- In the first rating set each service is randomly assigned with a service area. Ten of the services had a service area of $5 \mathrm{~km}$ around their location, 40 services were added with a service area of $3 \mathrm{~km}$ around their location and the remaining 50 services have a service area of $2 \mathrm{~km}$. The ratings for the services were chosen from a five point scale, where 1 represents dislike and 5 represents like. For each of the first 500 users an average of 20 ratings were added where the services were selected randomly and the rating was picked randomly as a value between 1 points to $5 \cdot \exp \left(-\frac{1}{2} \times \frac{\left\|x_{i}-x_{u}\right\|_{2}^{2}}{\sigma^{2}}\right)$ points.
- The second rating set was created to depict clustered user groups within the users. The assumed behaviour of these groups differs from the other users in having different preferences according to the distance to certain services. All in all ten such groups were created, with six groups consisting of ten users each, two groups with 20 users each and two groups with 50 users each. The number of services in which the behaviour of the users coincides was either five, ten or 20 services, the service area either $3.0 \mathrm{~km}$ or $5.0 \mathrm{~km}$. All service/user combinations not covered by this allocation were set to a service area of $2 \mathrm{~km}$. When creating the user groups and the according set of services, duplicate picks were allowed, so an user can be in more than one group and a service can be assigned to more than one group. If a service/user combination is covered by more than one user group, the service area assigned to the group created later was favoured. Furthermore the ratings in this rating set were calculated as described before.

Additionally in both data sets 25 users were added as noise users, also with a mean of 20 ratings and random ratings between 1 and 5 points.

\subsection{Evaluation Experiments}

To evaluate the efficiency of the proposed algorithms, we run several checks with both data sets against different metrics as suggested by Herlocker et al. [8]. Comparing the advantages and disadvantages of each metric with the requirements of our recommender system, we choose the following metrics for comparison: Mean Absolute Error, Mean Square Error, Pearsons Correlation per User and Pearsons 


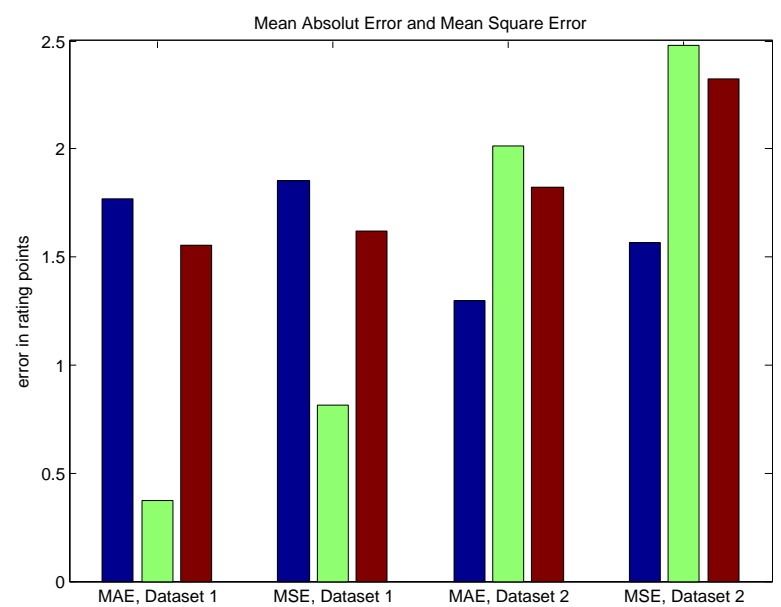

Figure 2. Mean absolute error and mean square error results of a conventional recommender algorithm (left, blue); geographical recommender 1 (center, green); geographical recommender 2 (right, red) for both data sets.

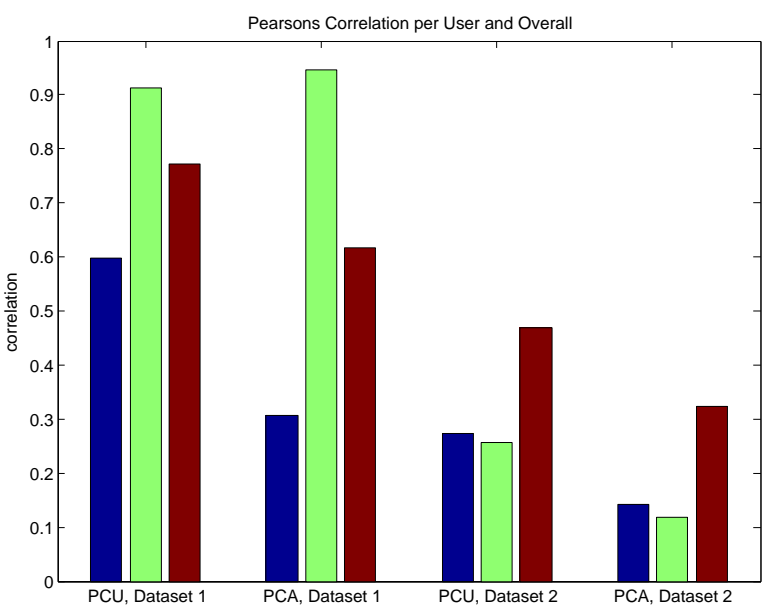

Figure 3. Pearsons correlation per user and absolute of a conventional recommender algorithm (left, blue); geographical recommender 1 (center, green); geographical recommender 2 (right, red) for both data sets.
Correlation Absolute. At the current stage of research we could not determine which items can be considered relevant or nonrelevant to the future user group. Therefore other metrics suggested by Herlocker et al. like the area under ROC-curves were not used for validation.

We assumed the "correct" prediction to be the expectation value of a standard distribution around the service with the given service area as standard variation at the location of the user. As this prediction is available for both data sets and any combination of users and services, we were able to run the metrics over all of the first 500 users and all services. Recommendations were calculated using the algorithms described in equations (1), (4) and (7) using a Pearsons correlation approach as similarity measure between the users.

\section{Results}

The test runs described in the previous section confirmed that both algorithms which include geospatial information into predicting a recommendation can adapt better to our data sets than the conventional recommender algorithm.

The mean absolute errors of the test runs with data set 1 are displayed on the left side in Figure 2. The non-spatial recommender system had an error of 1.7718 , which could be decreased to 0.3642 with the algorithm according to equation (4) and to 1.5514 with the algorithm according to equation (7) respectively. The same trend is observable in the mean squared errors (second group of bars in Figure 2). Here the error decreased from 1.8533 to 0.7849 and 1.6204 respectively. It was observed that none of the algorithms predicted ratings over 3.5. This might be due to the high amount of low ratings in the synthesised data set. Methods to compensate this behaviour like adjustment to the mean rating of users were not implemented as we just wanted to demonstrate the ability of the algorithms to adapt to spatial dependencies.

The correlation metrics, displayed in Figure 3 are not prone to such an offset in the predicted ratings. The Pearsons Correlation per user (left side of Figure 3) calculated the correlation between all predicted ratings of a user to the expected ratings, the value displayed is the mean value of all per user correlation. The correlation of 0.5989 from the conventional recommender algorithm was increased to 0.9095 with the algorithm according to equation (4) and to 0.7731 with the algorithm according to equation (7) respectively. The correlation of all ratings from all users is displayed by the second group of bars in Figure 3. The correlation of 0.3068 increased to 0.9392 and 0.6171 for the two algorithms incorporating spatial information.

When using the second data set, most results were noticeably worse than with data set 1 . This is most probably due to the more complex user behaviour simulated within this data set. In contrast to the results of the first data set, the best results regarding the error metrics were achieved with the non-spatial recommender. The mean square error was determined to be 1.2976 for the non-spatial recommender, 2.0121 for the spatial recommender according to equation (4) and 1.8245 for the spatial recommender according to equation (7). The results can be seen in the third group of bars in Figure 2. Respectively the mean absolute 
error was determined to be $1.5678,2.4813$ and 2.3247 for the three recommender systems, as can be seen in the right group of bars in Figure 2.

Regarding the correlation measures, the non-spatial recommender and the spatial recommender according to equation (4) performed both nearly equally well, with the nonspatial recommender providing slightly better results. In contrast, the spatial recommender according to equation (7) was able to provide results which were significantly better than the results of the other two recommender approaches. For the Pearsons Correlation per User (third set of bars in Figure 3 the results were 0.2726 for the non-spatial recommender system and 0.2557 and 0.4689 for the two spatial recommender system according to equations (4) and (7). The results of Pearsons Correlation over all data was even more significant: The non-spatial recommender had a correlation of just 0.1414 and the spatial recommender according to equation (4) of 0.1188 , while the spatial recommender according to equation (7) achieved a correlation of 0.3227 . These results are depicted in the right set of bars of Figure 3 .

\section{Discussion}

The considerably better results of the algorithm according to equation (4) when using data set 1 show that the algorithm is able to adapt to basic user preferences without distinct differences between the users. As already predicted in [15] these advantages dilute if more complex user behaviour is present, as within our data set 2 . Here the results of the first spatial aware algorithm perform only as good as the non-spatial recommender system.

To combine the advantages of both spatial aware recommender approaches a hybridisation seems to be advisable. Regarding Burke's classification of hybridisation approaches [6], either weighted or cascaded hybrid recommender systems seem to be applicable. In a weighted hybrid recommender system, the algorithm according to equation (4) can improve the results in early stages, when no or little user group behaviour can be predicted from the user ratings. In later stages the results of a recommender system according to equation (7) can improve the results by adapting to user group behaviour. By further extending this approach by the extension to the first algorithm as proposed in [14], the Ramp-Up problems (as discussed in the following section) might further be extenuated.

A cascaded hybridisation of the two approaches discussed in this paper might select services from a larger scale by using the algorithm according to equation (4), which are then further improved by the algorithm according to equation (7). This can be helpful to reduce computing time when a spatial recommender system is used in a wide area and with a mixture of services that are only interesting locally and services with a larger service area.

\section{Spatial Ramp-Up Problems}

It's well known that recommender systems need a certain amount of data to give beneficial recommendations. This is called the Jump-Starting [10] or Ramp-Up [6] effect. Besides the ramp-up in the starting phase of the system this applies to new users as well as to new items added to system. When these effects are handled properly, systems gain a crucial amount of usefulness.

When considering spatial recommendations, related effects can occur in addition to the before mentioned. By addressing these effects, the advantage of spatial aware recommender systems over conventional systems can be even higher:

- Moving User: In the operation of a spatial aware recommender system it might occur that the location of a user changes at a certain time. For instance, when the user moves from city A to city B, he will expect to get adequate recommendations for city $\mathrm{B}$ but the system only knows how his behaviour was in city A. Instead of learning the habits of the user again from scratch, spatial aware recommender systems should be able to find out if the user is willing to accept longer ways into the old, well known surrounding. Otherwise it should be able to find new items that can fill the gap of the items the user liked in the old surrounding.

- New Area: Additional to the problem of new items and new users in the system, in spatial aware recommendation systems deploying the system into new areas can evoke additional problems. Neither information about the area an item will cover nor about the interests of the users preferences are known. Here an item-based recommendation concept might help to predict the area a service will cover based on knowledge from similar services from other areas. Thus the system needs less time to provide reliable recommendations.

\section{Related Works}

Recommender systems have been the topic of a large amount of research. Comprehensive reviews of existing approaches and discussion of their pros and cons have been given for instance by Adomavicius and Tuzhilin [2] or Burke [6].

Burke's Chicago Entree restaurant recommender $[5,6,7]$ transported knowledge from one spatial domain to a new one: The system gave recommendations of Chicago restaurants, based on restaurants from other US cities the user liked. But this information was mainly used to find a similar restaurant in Chicago as a starting point based on similarity of the restaurants features. 
Until recently, only few works known to the authors included spatial information into recommender systems. These works emerge from the systems COMPASS by van Setten et al. [18], PILGRIM by Brunato and Battiti [4] and GeoWhiz by Horozov et al. [9]. In the last years more and more publications on this topic are published, mainly due to the increasing popularity of mobile internet and the associated applications. One example of these works is the system of Matyas and Schlieder described in [12].

COMPASS is a system based on mobile clients recommending Points of Interest (POIs) to tourists. The recommender system is a hybrid approach incorporating different prediction strategies, but none of them considers spatial information comparable with our approach.

The restaurant recommendation system GeoWhiz incorporates the distance between the residences of the users to implement a kind of demographic filtering. The selection of the items to be recommended is simply determined by a user setting. Thereby it is not possible to differentiate between items "worth a walk" and items that are only interesting if you are nearby.

The PILGRIM system is most similar to our approach; their ellipse fitting is comparable to our first algorithm. Both approaches disregard the fact that different user groups might have different preferences of the acceptable distance to a recommended item. This is addressed by the second algorithm proposed in this paper.

Matyas and Schlieder use geographical information associated with photographs available in online community portals. By creating a heatmap-model of geographical places they implement a user-based recommender system which is able to transport information about user preferences to new locations.

Furthermore, spatial information could be regarded as a context-dimension as proposed by Adomavicius et al. in [1]. But this work names the location just as a possible part of the user context, but does not give an approach of how to incorporate geographical information into the recommender algorithm.

\section{Future Work}

The proposed methods constitute one part of current research in the project WEITBLICK. The aim of the project is to provide elderly people with an assistance system for finding and making use of a diverse range of services. Such services include amongst others recreational and educational services, care and medical services as well as housekeeping services [16].

Currently requirements analysis on the user side [11] and conceptual design of hard- and software modules [13] are undertaken. The recommender system will be one of the main modules in the system and will be used to provide ser- vice recommendations according to the customer's needs. The user group targeted by the system includes self independent elderly people as well as residents of care homes with diverse physical and psychic limitations. Within the project mainly elderly people living in one city will be targeted. Nevertheless the ability to deploy the system into a wider area is an intended functionality.

In addition to this application, well implemented spatial recommender systems can be utilised in a wide range of other applications. In our case we assume the location of the user to be known and constant, but spatial recommendations are also realisable for mobile users. First of all location based services on mobile clients will benefit from such systems. For instance an electronic tourist guide might select interesting landmarks according to the personal interests of the user and the distance he is willing to walk to certain landmarks. This improves most current approaches which mainly use fixed distances to decide whether an item should be considered for recommendation or not.

Main goal for our research group is the implementation of a comprehensive recommender system for service relaying within the WEITBLICK project as described in the previous section. Thereby it will be possible to evaluate the proposed methods with real world data as well and get more realistic results of how well the algorithms work.

Further enhancements of the algorithms will address the problems described in section 6 to make the application of our recommender system more scaleable in real world operation. The possible hybridisation discussed in section 5 will be implemented to improve quality. As all the results within this paper are only achieved by using synthesised data sets, test runs with real world data have to be made. Thereby it has to be revised whether the underlying assumptions of user behaviour are valid or not.

So far only the euclidean distance between users and items is taken into consideration. But other distance measures might be incorporated into the system easily. In the past our research group developed a route planning algorithm [17] which adapts to abilities and handicaps of (disabled) persons. This algorithm estimates the energy costs for the best way between two locations. It is expected that using this energy costs instead of simple euclidean distance will favor items that are easier to reach for the user over items with worse accessibility.

\section{Conclusions}

We presented an approach to add spatial awareness to recommender systems. Although no real world data was available to evaluate the algorithm, tests with synthesised data sets showed promising results. So far only basic methods for recommender systems were used to show the idea of the concept. 
When the proposed method is further augmented with other well known approaches to recommender systems, it is most likely to benefit from recognising spatial relations between users and items. This might become quite useful in applications of location based services and other domains where local spatial relations influence the significance of an item to the user.

\section{Acknowledgements}

The results presented in this paper emerged from the WEITBLICK project, funded by the German Bundesministerium für Bildung und Forschung (BMBF) under Grant ID 01FC08029. The authors would like to thank all involved partners from the project for their support.

\section{References}

[1] G. Adomavicius, R. Sankaranarayanan, S. Sen, and A. Tuzhilin. Incorporating contextual information in recommender systems using a multidimensional approach. ACM Transactions on Information Systems, 25(1):103-145, 2005.

[2] G. Adomavicius and A. Tuzhilin. Toward the Next Generation of Recommender Systems: A Survey of the Stateof-the-Art and Possible Extensions. IEEE Transactions on Knowledge and Data Engineering, 17(6):734-749, June 2005.

[3] A. Asuncion and D. J. Newman. UCI Machine Learning Repository, 2007.

[4] M. Brunato and R. Battiti. PILGRIM: A Location Broker and Mobility-Aware Recommendation System. In Proceedings of the First IEEE International Conference on Pervasive Computing and Communications, 2003.

[5] R. Burke. The Wasabi Personal Shopper: A Case-Based Recommender System. In Proceedings of the 11th Conference on Innovative Applications of Artificial Intelligence, pages 844-849, 1999.

[6] R. Burke. Hybrid Recommender Systems: Survey and Experiments. User Modeling and User-Adapted Interaction, 12:331-370, 2002.

[7] R. D. Burke, K. J. Hammond, and B. C. Young. The FindMe Approach to Assisted Browsing. IEEE Expert, 12:32-40, 1997.

[8] J. L. Herlocker, J. A. Konstan, L. G. Terveen, and J. T. Riedl. Evaluating Collaborative Filtering Recommender Systems. ACM Transactions on Information Systems, 22(1):5-53, Jan. 2004.

[9] T. Horozov, N. Narasimhan, and V. Vasudevan. Using Location for Personalized POI Recommendation in Mobile Environments. In Proceedings of the 2006 Symposium on Applications and the Internet (SAINT'06), pages 124-129, 2006.
[10] J. A. Konstan, J. Riedl, A. Borchers, and J. L. Herlocker. Recommender Systems: A GroupLens Perspective. Recommender Systems: Papers from the 1998 Workshop. AAAI Technical Report WS-98-08, pages 60-64, 1999.

[11] S. Lutherdt, C. Stiller, K. Lienert, S. Spittel, F. Roß, C. Ament, and H. Witte. Design of an assistance system for elderly based on analyses of needs and acceptance. Lecture Notes in Computer Science: Universal Access in Human-Computer Interaction. Proc. of the 5th Int. Conference UAHCI 2009, held as part of HCI International 2009, San Diego, CA, USA, pages 96-105, July 2009.

[12] C. Matyas and C. Schlieder. A Spatial User Similarity Measure for Geographic Recommender Systems. Lecture Notes in Computer Science: GeoSpatial Semantics: Third International Conference, 5892/2009:122-139, 2009.

[13] K. Renhak, C. Stiller, H.-P. Schade, J. Seitz, E. Schön, F. Roß, C. Ament, H. Hildebrandt, and M. Oswald. WEITBLICK - Wissensbasierte Technologien und bedarfsgerechte Leistungen für Senioren durch individualisierte CareKonzepte. In Technologienutzung ohne Barrieren: Zusammenfassung der Beiträge zum Usability Day VII, pages 164169, Mar. 2009.

[14] C. Stiller, F. Roß, and C. Ament. Ramp-Up-Effects in Spatial Aware Recommender Systems. In Proceedings of the 4th International Conference on Ubiquitous Information Technologies and Applications - ICUT'09, pages 171-175, Dec. 2009.

[15] C. Stiller, F. Roß, and C. Ament. Towards Spatial Awareness in Recommender Systems. In Proceedings of the 4th International Conference for Internet Technology and Secured Transactions - ICITST'09, Nov. 2009.

[16] C. Stiller, F. Roß, C. Ament, K. Renhak, J. Seitz, E. Schön, H.-P. Schade, H. Hildebrandt, and M. Oswald. WEITBLICK - Infrastruktur für eine bedarfsgerechte Dienstleistungsvermittlung. In 2. Deutscher AAL-Kongress 2009, Jan. 2009.

[17] C. Stiller, F. Roß, R. Rost, M. Stein, and D. Karimanzira. A Framework for Individualized Route Planning and Navigation for Pedestrian Users. In Proc. of 51st International Scientific Colloquium, IWK, pages 15-16. TU Ilmenau, Sept. 2006.

[18] M. van Setten, S. Pokraev, and J. Koolwaaij. Context-Aware Recommendations in the Mobile Tourist Application COMPASS. Lecture Notes in Computer Science: Adaptive Hypermedia and Adaptive Web-Based Systems, 3137/2004:235244, 2004. 